\title{
cosmetics
}

ISSN 2079-9284

www.mdpi.com/journal/cosmetics

Article

\section{The Scalp Has a Lower Stratum Corneum Function with a Lower Sensory Input than Other Areas of the Skin Evaluated by the Electrical Current Perception Threshold}

\author{
Yutaka Takagi *, Hiroko Takatoku, Hiroyuki Terazaki, Tadashi Nakamura, Koichi Ishida and \\ Takashi Kitahara \\ Kao Corporation, R\&D, 2-1-3 Bunka, Sumida-ku, Tokyo 131-8501, Japan; \\ E-Mails: takatoku.hiroko@kao.co.jp (H.Ta.); terasaki.hiroyuki@kao.co.jp (H.T.); \\ nakamura.tadashi@kao.co.jp (T.N.); ishida.koichi@kao.co.jp (K.I.); kitahara.takashi@kao.co.jp (T.K.) \\ * Author to whom correspondence should be addressed; E-Mail: takagi.yutaka@kao.co.jp; \\ Tel.: +81-3-5630-9792; Fax: +81-3-5630-9330.
}

Academic Editor: Enzo Berardesca

Received: 30 September 2015 / Accepted: 17 November 2015 / Published: 20 November 2015

\begin{abstract}
Many people feel frequent prickling or itching sensations on their scalp. The scalp is an atypical area of the skin since it is normally covered with thick hair and has many sebaceous glands and sweat glands. The scalp often has skin problems that can affect its sensitivity and functions. However, not much is known about stratum corneum function and the neural sensitivity of the scalp. Here we evaluated stratum corneum function and the neural sensitivity of the scalp of 47 normal male individuals in various skin conditions and compared the results to that to the forehead. The neural sensitivity was evaluated by measuring the electrical current perception threshold (CPT). The cutaneous barrier function and stratum corneum moisture-retention ability (MRA) of the scalp were significantly lower than on the forehead, even if there were some scalp problems. Depending on the increase in severity of scalp skin problems, both these skin functional properties and the CPT decreased significantly. However, regardless of its lower functional properties, scalp skin was not significantly lower than that of the forehead. Although the scalp has a low stratum corneum function compared with the forehead and has easily induced skin problems, the scalp skin has less sensitive sensory nerves, resulting in experiencing a worsening of scalp symptoms more easily.
\end{abstract}


Keywords: scalp skin; stratum corneum; current perception threshold; forehead

\section{Introduction}

The scalp is a peculiar type of skin that is normally covered with thick hair and has many sebaceous glands and sweat glands. Those special circumstances may cause a prickling or itching feeling on the scalp, which is felt very often by some subjects. We have found that about one-third of people worry about these unpleasant feelings in Japan (data not shown). Furthermore, the scalp can have many additional problems, such as dandruff, odor, and so on. To take care of the scalp and hair, many hair care products, such as shampoos, hair conditioners, hair styling products, and drugs, are used almost daily. There are many reports about dandruff [1-4]. Bin Saif reviewed scalp pruritus, the nerves on hair follicle and hair, and mediators and receptors involved in the itchy sensation [5]. However, there are few reports about the stratum corneum functions [4-7] or the sensitivity of the scalp [8-12] compared with skin at other body regions. Furthermore, the evaluations of actual nerve reactions compared with other body regions are very few. In this study, we analyzed the skin's functional properties and the neural sensitivity of the scalp. The electrical current perception threshold (CPT) of sensory nerves measured with a Neurometer ${ }^{\circledR}$ has been used to demonstrate abnormalities in neuropathic conditions [13-15] and also to evaluate sensitive skin [16]. The sensitivity of the scalp to electric stimulation using a Neurometer ${ }^{\circledR}$ was compared to the forehead of each subject. Furthermore, we analyzed the relationship between skin properties and neural sensitivity and various scalp conditions, such as erythema, scaling, papules, and so on. From these analyses we found that the scalp has a low stratum corneum function compared with the forehead and has easily induced skin problems; however, the scalp skin has fewer sensitive sensory nerves, resulting in experiencing a worsening of scalp symptoms more easily.

\section{Experimental Section}

\subsection{Study Design}

The present study adhered to the tenets of the Declaration of Helsinki. All clinical studies were reviewed and approved by the Review Board of the Kao Corporation (Tokyo, Japan) and formal informed consent was obtained from each subject before the study.

\subsection{Participants}

Healthy male subjects were recruited randomly and 47 male volunteers participated in this study. Subjects with alopecia areata, severe scalp eczema, and/or severe dandruff were excluded.

\subsection{Test Procedure}

Each subject washed his head and face on the eve of the evaluation day. Following this washing, topical application of skin care products on the analyzed areas was prohibited until after the skin 
evaluation. Both sides of the head (a $5 \mathrm{~mm} \times 5 \mathrm{~mm}$ area 5 to $7 \mathrm{~cm}$ above the ear) were used for the evaluation. The hairs of the evaluated areas were removed by scissors immediately before the evaluation. Sebum levels on the forehead and the scalp were analyzed with a Sebusheet ${ }^{\circledR}$ ASS-001 (Asahi Techno Lab. Ltd., Kanagawa, Japan) according to seven grades (0, 25, 50, 75, 100, 150, and $200 \mu \mathrm{g} / \mathrm{cm}^{2}$ ). A TPX-90 Handy PH meter (Tokyo Glass Kikai Ltd., Tokyo, Japan) was used with a flat glass electrode according to EEMCO guidance [17]. The average value of three measurements was used for analysis. The CPT was measured with an electric current sine wave stimulator of sensory nerves (Neurometer ${ }^{\circledR} \mathrm{CPT} / \mathrm{C}$, Neurotron Inc., Baltimore, MD, USA). Three sine wave stimulations (at 2000, 250 , and $5 \mathrm{~Hz}$ ) produced by the Neurometer ${ }^{\circledR}$ provided selective simulations for three subsets of nerve fibers [15]. Following these measurements, the head and forehead of each subject was washed with a cleanser and dried by a specialist. After acclimation at $20 \pm 2{ }^{\circ} \mathrm{C}, 40 \% \pm 5 \%$ humidity for 20 min, transepidermal water loss (TEWL) and stratum corneum moisture-retention ability (MRA) were measured with a Tewameter TM210 ${ }^{\circledR}$ (Courage + Khazaka electronic GmbH, Cologne, Germany) and a Corneometer ${ }^{\circledR}$ CM825 (Courage + Khazaka electronic $\mathrm{GmbH}$ ), respectively. Specialists who were internally trained visually rated the scaling, erythema, papules, eschars, pustules, and nodules on a scale of 0 to $4(0=$ none, $1=$ slight, $2=$ mild, $3=$ moderate, and $4=$ severe $)$ on the evaluated areas of the scalp.

\subsection{Statistical Analysis}

Data obtained from both sides of the head were calculated as averages. Differences between the scalp and the forehead were determined statistically with Student's paired $t$-test.

\section{Results}

\subsection{Participants with Skin Symptoms}

Forty-seven males, aged 27 to 46 years old $(36.1 \pm 4.8$, mean \pm S.D.), were included in this evaluation. All subjects had hair on the evaluated areas and none of them had severe scalp problems. Their scalp conditions are summarized in Table 1.

Table 1. Scalp skin symptoms of subjects.

\begin{tabular}{ccccccc}
\hline Score & Scaling & Erythema & Papule & Eschar & Pustule & Nodule \\
\hline 0 & 15 & 3 & 28 & 35 & 31 & 43 \\
1 & 18 & 26 & 11 & 8 & 12 & 3 \\
2 & 11 & 14 & 8 & 4 & 1 & 1 \\
3 & 3 & 4 & 0 & 0 & 3 & 0 \\
4 & 0 & 0 & 0 & 0 & 0 & 0 \\
SUM & 47 & 47 & 47 & 47 & 47 & 47 \\
\hline
\end{tabular}




\subsection{Comparison of Measured Data between the Scalp and the Forehead}

Prior to washing the scalp and the face, the sebum level of the scalp was more than $50 \%$ higher than that of the forehead (scalp: $89 \pm 29 \mu \mathrm{g} / \mathrm{cm}^{2}$, forehead: $59 \pm 31 \mu \mathrm{g} / \mathrm{cm}^{2}, p<0.01$, Figure 1A). However, the average skin surface $\mathrm{pH}$ of the scalp was similar to that of the forehead (scalp: $4.80 \pm 0.45$, forehead: $4.80 \pm 0.40$, Figure 1B).

Following washing with the cleanser, the MRA of the scalp was significantly lower than that of the forehead (scalp: $34.5 \pm 9.4$, forehead: $54.3 \pm 11.2, p<0.01$, Figure 1C) and the TEWL of the scalp was significantly higher than that of the forehead (scalp: $6.6 \pm 1.6 \mathrm{~g} / \mathrm{m}^{2} \cdot \mathrm{hr}$, forehead: $5.5 \pm 1.6 \mathrm{~g} / \mathrm{m}^{2} \cdot \mathrm{hr}$, $p<0.01$, Figure 1D).

A

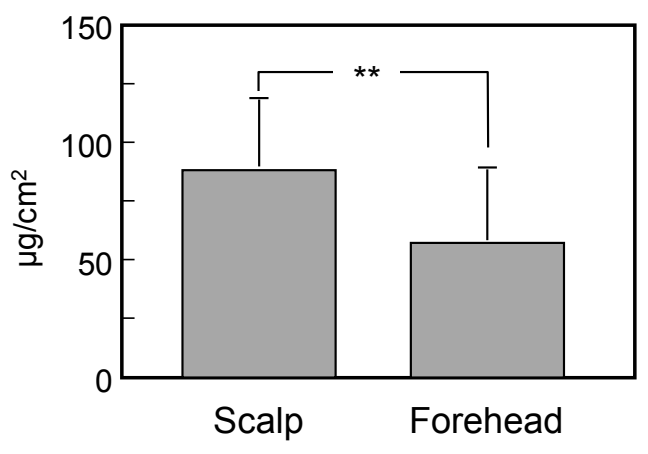

C

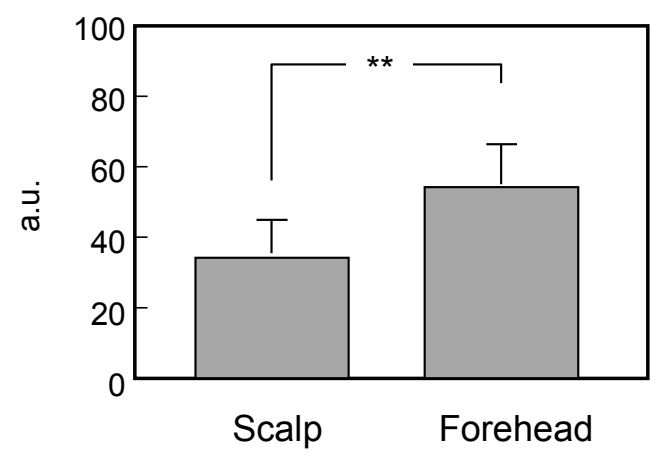

B

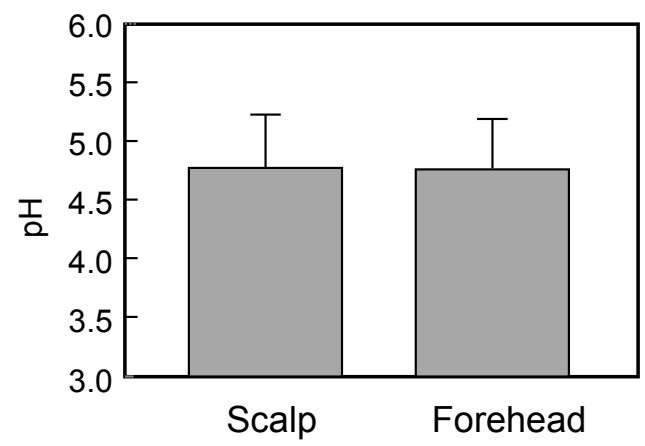

D

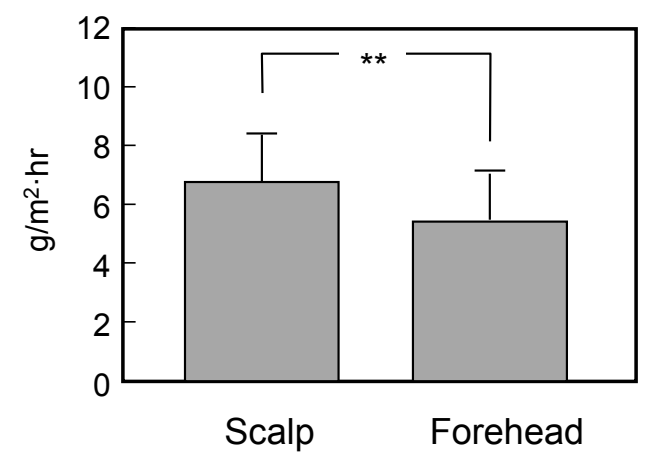

Figure 1. Comparison of measured data for sebum level (A), skin surface pH (B), MRA (C), and TEWL (D) between the scalp and the forehead. Each column indicates the average and the bars show standard deviation. $* *$ indicates $p<0.01$ as a significant difference.

The CPT on the scalp and the forehead was measured with a Neurometer ${ }^{\circledR}$ prior to washing with the cleanser. Although the MRA and TEWL of the scalp indicate that the scalp has a lower stratum corneum function than the forehead, the scalp had a significantly higher CPT value than the forehead (Figure 2A-C). 
A

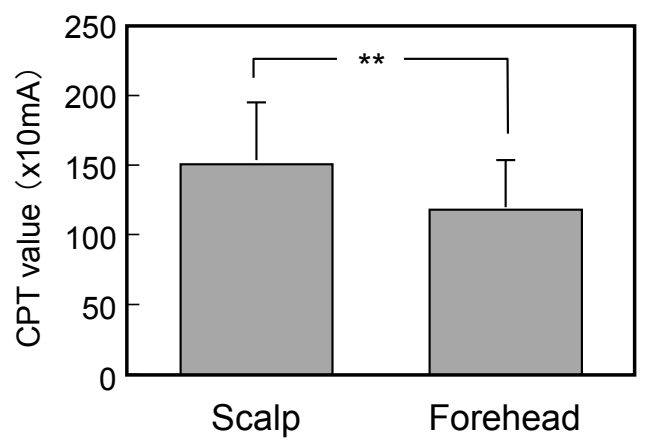

C

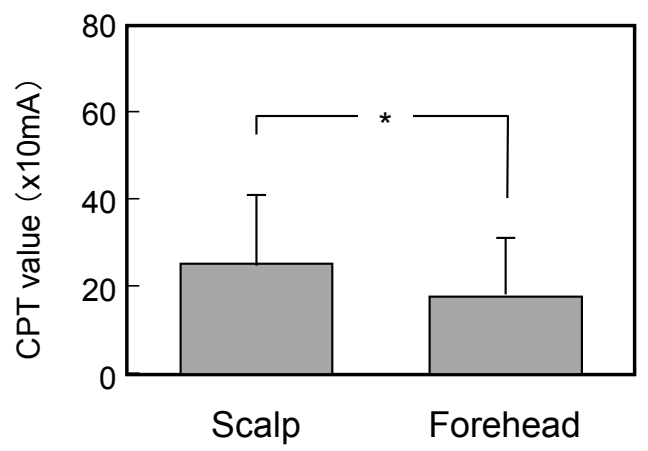

B

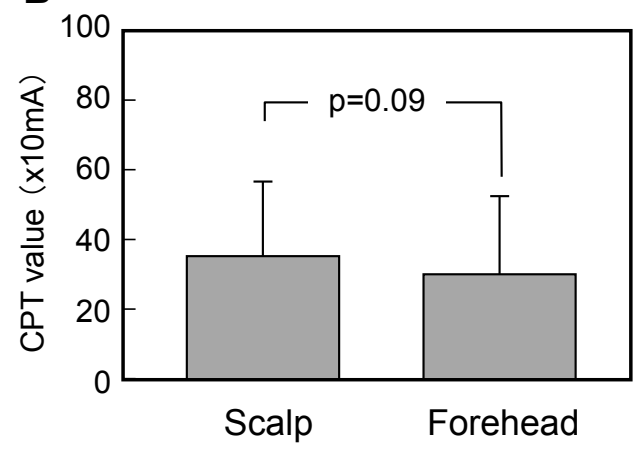

Figure 2. Comparison of measured data for CPT for $2000 \mathrm{~Hz}(\mathbf{A}), 250 \mathrm{~Hz}(\mathbf{B})$, and $5 \mathrm{~Hz}(\mathbf{C})$ between the scalp and the forehead. Each column indicates the average and the bars show standard deviation. * indicates $p<0.05$ and $* *$ indicates $p<0.01$ as a significant difference.

\subsection{Comparison of Measured Data between Scalps with Skin Symptoms of Different Severity and the Forehead}

The properties of the stratum corneum and the cutaneous sensitivity might be affected by the skin symptoms. Thus, we analyzed the relationship between the stratum corneum properties and the severity of skin symptoms (as shown in Table 1) and found that every skin symptom affected the stratum corneum properties and the cutaneous sensitivity with the same tendency (data not shown). Because of the low severity of each type of skin symptom, the subjects were classified into three groups according to the severity of the scalp skin problem. Group I did not have any kind of skin symptoms with a severity score of more than $1(n=21)$, Group II had one skin symptom with a severity score of more than $1(n=12)$, and Group III had more than one skin symptom with a severity score of more than $1(n=14)$.

Depending on the increase in the severity of scalp skin problems, the sebum level decreased significantly, but even Group III had more sebum on the scalp than on the forehead (Figure 3A). The skin surface $\mathrm{pH}$ was significantly higher with the increased severity of skin problems (Figure 3B). The MRA of the scalp of Group I was significantly lower than that of the forehead and was dependent on the increased severity of skin problems (Figure 3C). The TEWL of the scalp of Group I was significantly higher than that of the forehead, but it was even higher in Groups II and III (Figure 3D). 
A

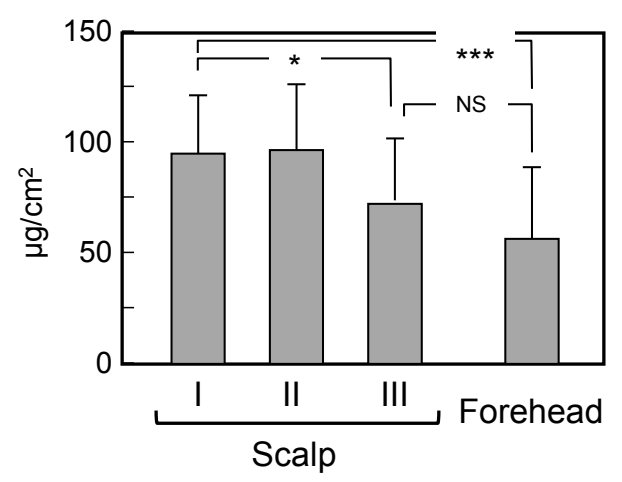

C

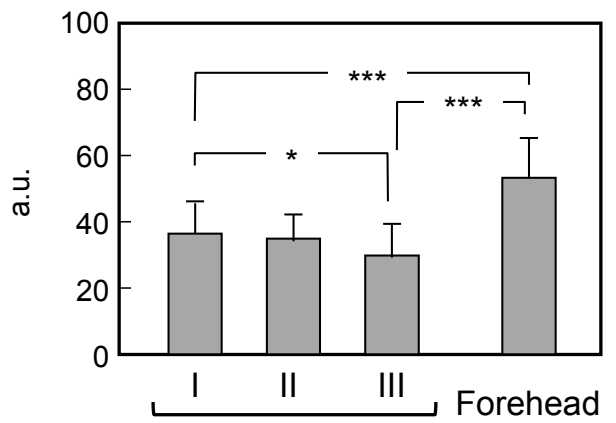

B

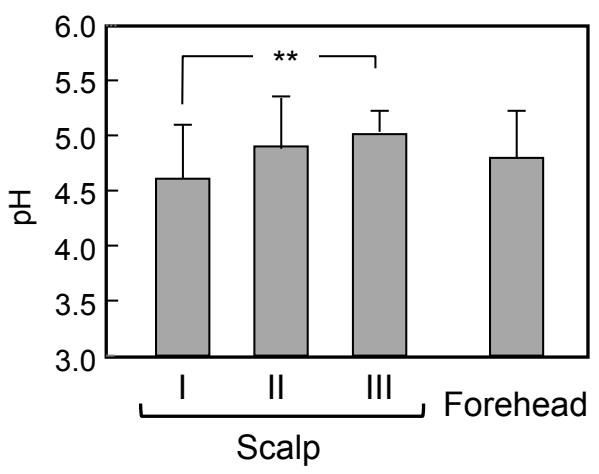

D

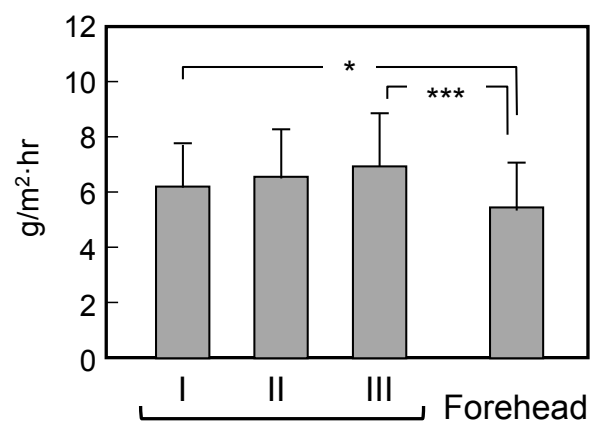

Figure 3. Comparison of measured data for sebum level (A), skin surface pH (B), MRA (C), and TEWL (D) between scalps with skin symptoms of different severity and the forehead. Each column indicates the average and the bars show standard deviation. * indicates $p<0.05, * *$ indicates $p<0.01$ and *** indicates $p<0.001$ as a significant difference. NS indicates as no significant difference.

The CPT on the scalp of Group I was significantly higher than that of the forehead at each level of current. Depending on the increased severity of skin problems, these values decreased significantly in Groups II and III. Only the $2000 \mathrm{~Hz}$ current had a higher CPT value on the scalp of Group III than on the forehead and there was no significant difference in the CPT value for 250 and $5 \mathrm{~Hz}$, even in Group II (Figure 4A-C).

A

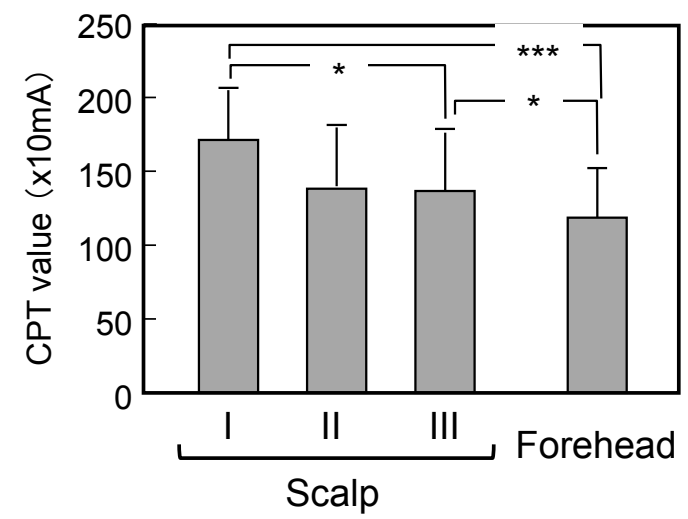

B

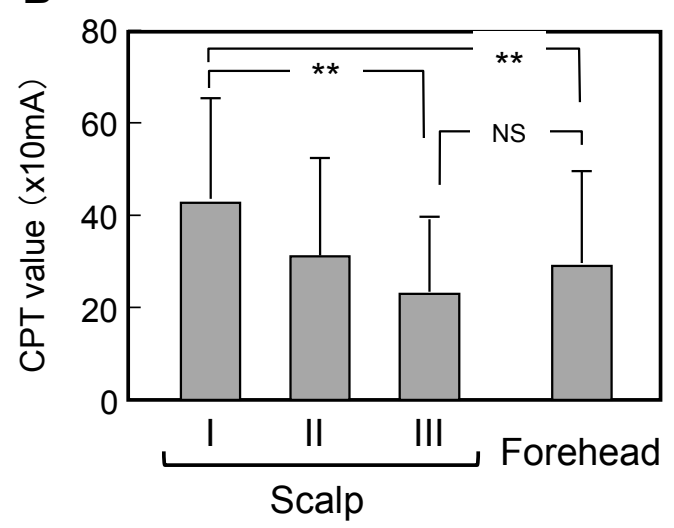

Figure 4. Cont. 


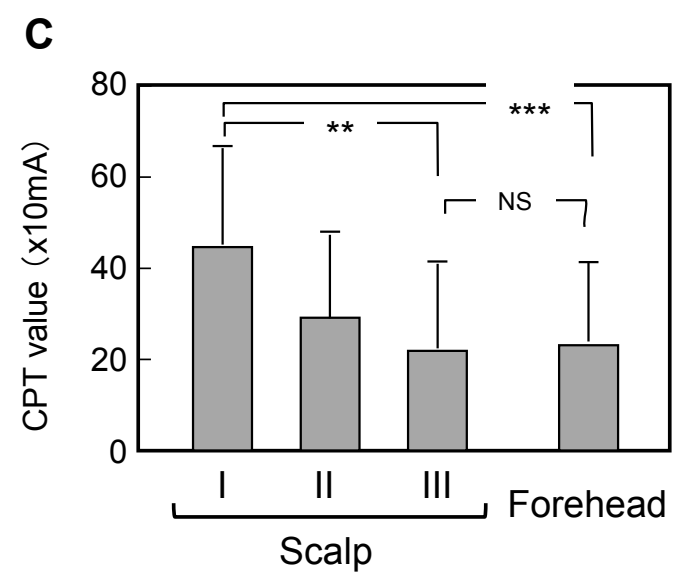

Figure 4. Comparison of measured data for CPT for $2000 \mathrm{~Hz}(\mathbf{A}), 250 \mathrm{~Hz}(\mathbf{B})$, and $5 \mathrm{~Hz}(\mathbf{C})$ between scalps with different skin symptoms and the forehead. Each column indicates the average and the bars show standard deviation. * indicates $p<0.05, * *$ indicates $p<0.01$ and $* * *$ indicates $p<0.001$ as a significant difference. NS indicates as no significant difference.

\section{Discussion}

Although scalp skin is less sensitive than other areas of skin, there is a high prevalence of pruritus. Furthermore, the scalp easily develops other skin problems, not only pruritus but also dandruff, odor, and itching, because it has many sweat glands and sebaceous glands and is usually covered with thick hair. However, there are only a few reports about stratum corneum function on the scalp. O'goshi et al. reported that the scalp has a significantly higher high-frequency conductance and a significantly lower TEWL than the cheek [7]. However, in our evaluation, the scalp has a significantly lower capacitance and a significantly higher TEWL compared to the forehead. As the skin surface hydration of the cheek is significantly lower than that of the forehead [18], it is presumed that both the scalp and the cheek have a lower water-holding capacity compared to the forehead. The lower conductance of the cheek might be caused by a low sebum level [18], but our evaluation showed that the scalp has significantly more sebum compared to the forehead. In our evaluation, TEWL and MRA were measured following skin washing with cleansers and this treatment might cause the different results between these two evaluations. Additionally, the scalp conditions and hair affect these values, e.g., all subjects in our evaluation had thick hair on the side of the head that was evaluated. Stratum corneum functions such as TEWL and MRA are greatly affected by the existence of water such as sweat and sebum. The amount of sweat and sebum are changes dependent on the subjects' condition prior to the evaluation. Thus, to standardize the condition, the stratum corneum functions were evaluated following washing with cleanser and habituation in this evaluation.

Despite its low stratum corneum functions, the scalp has a higher CPT value which indicates that the scalp is more sensorily obtuse compared to the forehead. Mori et al. reported that decreases in skin surface hydration and increases in TEWL are correlated with the decrease in CPT on the forearm [19]. Also, Tominaga et al. reported that dry skin induced by acetone treatment had increased intraepidermal nerve fibers in mice [20]. The lower stratum corneum functions such as sensitive skin and atopic dermatitis can easily cause the skin inflammation and pruritus. Moreover, the thicker epidermis [21] and greater number of stratum corneum layers [6] of the scalp compared to the forehead may affect the 
sensitivity. However, the scalp skin has less sensitive sensory nerves, even if the stratum corneum functions are low, showing that the scalp is a very special property. The high humidity which leads to sweat and sebum, resulting in a great deal of moisture on the stratum corneum, might be one reason causing these less sensitive sensory nerve. Another evaluation indicated that the scalp is notably insensitive to innocuous and noxious changes in temperature [10]. Moreover, it was reported that scalp skin is less sensitive to histamine-induced experimental itching compared to the forearm [12], although the scalp was more sensitive to a sodium lauryl sulphate challenge than the forearm [9].

The CPT of sensory nerves measured with a Neurometer ${ }^{\circledR}$ has been used to demonstrate abnormalities in neuropathic conditions [13-15]. Five Hz stimulates unmyelinated $\mathrm{C}$ fibers, resulting in a slow pain described as "burning" or "stinging", $250 \mathrm{~Hz}$ stimulates Ad fibers with a feeling of fast pain and temperature, and $2000 \mathrm{~Hz}$ stimulates $\mathrm{Ab}$ fibers producing the sensations of touch and a more mechanical quality.

In our evaluation, normal scalp skin was significantly less sensitive against all three of these electrical stimuli compared to the forehead; these results agree well with the previous reports described above. However, with increased skin problems and skin surface conditions, the scalp became more sensitive, almost at the same level as the forehead in Group III, which has relatively severe scalp problems, especially for 250 and $5 \mathrm{~Hz}$ stimuli.

The scalp has low stratum corneum functions even under conditions that readily induce skin problems, such as high humidity and rich sebum levels which result in the existence of many microbes such as Malassezia. However, because of its low neural sensitivity, it might be easy to worsen the skin problems on the scalp. It is presumed that such severe skin circumstances make the scalp insensitive, but increase the severity of the skin problems. Saint-Martory et al. reported that $36.2 \%$ of French subjects say they suffer from sensitive scalp skin [22]; however, there are very few reports about sensitive scalp skin [23,24]. Our study shows that, although the scalp has a low stratum corneum function compared with to the forehead, the scalp skin has less sensitive sensory nerves. Therefore, scalp skin might have easily induced skin problems and be more prone to worsening compared to other regions of skin.

\section{Conclusions}

The scalp skin has less sensitive sensory nerves, despite its low stratum corneum function compared to that of the forehead. Thus, the scalp skin can suffer from easily induced skin problems and more adequate care should be necessary for maintaining healthy scalp skin.

\section{Acknowledgments}

This study was supported financially by the Kao Corporation. We thank our colleagues in the Global R\&D, Kao Corporation for their kind help during the study. 


\section{Author Contributions}

Hiroko Takatoku, Takashi Kitahara, Hiroyuki Terazaki, Tadashi Nakamura and Koichi Ishida conceived and designed the experiments; Hiroko Takatoku and Hiroyuki Terazaki performed the experiments; Hiroko Takatoku and Yutaka Takagi analyzed the data; Yutaka Takagi wrote the paper.

\section{Conflicts of Interest}

No conflict of interest by disclosing any financial arrangements in this paper. All authors are employees of Kao Corporation and this study was supported financially by Kao Corporation.

\section{References}

1. Hay, R.J. Malassezia, dandruff and seborrhoeic dermatitis: An overview. Br. J. Dermatol. 2011, $165,2-8$.

2. Turner, G.A.; Hoptroff, M.; Harding, C.R. Stratum corneum dysfunction in dandruff. Int. J. Cosmet. Sci. 2012, 34, 298-306.

3. Gaitanis, G.; Magiatis, P.; Hantschke, M.; Bassukas, I.D.; Velegraki, A. The Malassezia genus in skin and systemic diseases. Clin. Microbiol. Rev. 2012, 25, 106-141.

4. Warner, R.R.; Schwartz, J.R.; Boissy, Y.; Dawson, T.L., Jr. Dandruff has an altered stratum corneum ultrastructure that is improved with zinc pyrithione shampoo. J. Am. Acad. Dermatol. 2001, 45, 897-903.

5. Bin Saif, G.A.; Ericson, M.E.; Yosipovitch, G. The itchy scalp-Scratching for an explanation. J. Eur. Exp. Dermatol. 2011, 20, 959-968.

6. Ya-Xian, Z.; Suetake, T.; Tagami, H. Number of cell layers of the stratum corneum in normal skin-relationship to the anatomical location on the body, age, sex and physical parameters. Arch. Dermatol. Res. 1999, 291, 555-559.

7. O'goshi, K.; Iguchi, M.; Tagami, H. Functional analysis of the stratum corneum of scalp skin: Studies in patients with alopecia areata and androgenetic alopecia. Arch. Dermatol. Res. 2000, 292, 605-611.

8. Myles, K.; Kalb, J.T.; Lowery, J.; Kattel, B.P. The effect of hair density on the coupling between the tactor and the skin of the human head. Appl. Ergon. 2015, 48, 177-185.

9. Zhai, H.; Fautz, R.; Fuchs, A.; Bhandarkar, S.; Maibach, H.I. Human scalp irritation compared to that of the arm and back. Contact Dermatitis 2004, 51, 196-200.

10. Essick, G.; Guest, S.; Martinez, E.; Chen, C.; McGlone, F. Site-dependent and subject-related variations in perioral thermal sensitivity. Somatosens. Mot. Res. 2004, 21, 159-175.

11. Mehrabyan, A.; Guest, S.; Essick, G.; McGlone, F. Tactile and thermal detection thresholds of the scalp skin. Somatosens. Mot. Res. 2011, 28, 31-47.

12. Rukwied, R.; Zeck, S.; Schmelz, M.; McGlone, F. Sensitivity of human scalp skin to pruritic stimuli investigated by intradermal microdialysis in vivo. J. Am. Acad. Dermatol. 2002, 47, 245-250.

13. Masson, E.A.; Veves, A.; Fernando, D.; Boulton, A.J.M. Current perception thresholds: A new, quick, and reproducible method for the assessment of peripheral neuropathy in diabetes mellitus. Diabetologia 1989, 32, 724-728. 
14. Baquis, G.D.; Brown, W.F.; Capell, J.T.; Chaudhry, V.; Cros, D.; Drexinger, B.; Gelblum, J.B.; Gilchrist, J.M.; Gitter, A.J.; Haig, A.J.; et al. Technology review: The Neurometer ${ }^{\circledR}$ Current Perception Threshold (CPT). Muscle Nerve 1999, 22, 523-531.

15. Kobayashi, H.; Kikuchi, K.; Tsubono, Y.; Tagami, H. Measurement of electrical current perception threshold of sensory nerves for pruritus in atopic dermatitis patients and normal individuals with various degrees of mild damage to the stratum corneum. Dermatology 2003, 206, 204-211.

16. Lee, E.; An, S.; Lee, T.R.; Kim, H.K. Development of a novel method for quantitative evaluation of sensory skin irritation inhibitors. Skin Res. Technol. 2009, 15, 464-469.

17. Parra, J.L.; Paye, M. EEMCO guidance for the in vivo assessment of skin surface $\mathrm{pH}$. Skin Pharmacol. Appl. Skin Physiol. 2003, 16, 188-202.

18. Tagami, H. Location-related differences in structure and function of the stratum corneum with special emphasis on those of the facial skin. Int. J. Cosmet. Sci. 2008, 30, 413-434.

19. Mori, T.; Ishida, K.; Mukumoto, S.; Yamada, Y.; Imokawa, G.; Kabashima, K.; Kobayashi, M.; Bito, T.; Nakamura, M.; Ogasawara, K.; et al. Comparison of skin barrier function and sensory nerve electric current perception threshold between IgE-high extrinsic and IgE-normal intrinsic types of atopic dermatitis. Br. J. Dermatol. 2010, 162, 83-90.

20. Tominaga, M.; Ozawa, S.; Tengara, S.; Ogawa, H.; Takamori, K. Intraepidermal nerve fibers increase in dry skin of acetone-treated mice. J. Dermatol. Sci. 2007, 48, 103-111.

21. Florence, P.; Cornillon, C.; D’arras, M.F.; Flament, F.; Panhard, S.; Diridollou, S.; Loussouarn, G. Functional and structural age-related changes in the scalp skin of Caucasian women. Skin Res. Technol. 2013, 19, 384-393.

22. Saint-Martory, C.; Roguedas-Contios, A.M.; Sibaud, V.; Degouy, A.; Schmitt, A.M.; Misery, L. Sensitive skin is not limited to the face. Br. J. Dermatol. 2008, 158, 130-133.

23. Misery, L.; Sibaud, V.; Ambronati, M.; Macy, G.; Boussetta, S.; Taieb, C. Sensitive scalp: Does this condition exist? An epidemiological study. Contact Dermatitis 2008, 58, 234-238.

24. Misery, L.; Rahhali, N.; Ambonati, M.; Black, D.; Saint-Martory, C.; Schmitt, A.M.; Taieb, C. Evaluation of sensitive scalp severity and symptomatology by using a new score. J. Eur. Acad. Dermatol. Venereol. 2011, 25, 1295-1298.

(C) 2015 by the authors; licensee MDPI, Basel, Switzerland. This article is an open access article distributed under the terms and conditions of the Creative Commons Attribution license (http://creativecommons.org/licenses/by/4.0/). 